\title{
Age Changes in Cerebellar Oligodendrocytes: The Appear- ance of Nuclear Filaments and Increase in the Volume Density of the Nucleus and in the Number of Dark Cell Forms*
}

\author{
Rogério A. F. Monteiro ${ }^{1}$, Luís E. C. Conceição ${ }^{1}$, Eduardo RoCHA ${ }^{1}$ and Mário M. MARINi-AbreU ${ }^{2}$ \\ Laboratory of Histology and Embryology ${ }^{1}$, and Laboratory of Neuroanatomy ${ }^{2}$, Institute of Biomedical Sciences, University of \\ Oporto, Porto, Portugal \\ Received September 21, 1994 ; revised February 21, 1995
}

\begin{abstract}
Summary. A qualitative and quantitative ultrastructural study (volume density of the nucleus and cell countings) on age-related changes occurring in neocerebellar oligodendrocytes of the granular layer was carried out in female albino rats aged 2-24 months. Taking into account the cytological features, two types of oligodendrocytes could be discerned, designated as Type I and Type II. Observations showed that, from 18 months onwards, the appearance of bundles of nuclear filaments running throughout the euchromatin areas are common occurrence in Type II oligodendrocytes. The lowest and highest mean volume density of the nucleus is achieved, respectively, at 2 months (50.67\%) and at 21 months $(64.93 \%)$; the analysis of variance of the parameter with ageing displays a most statistically significant result. There is also a positive and linear correlation between the volume density and age. The percentage of Type I oligodendrocytes (out of the total of counted oligodendrocytes) predominates from 2 to 15 months; after being even at 18 months, the percentage of Type II oligodendrocytes preponderates at 21 and 24 months. Although oligodendrocytes are capable of undergoing mitosis, it is concluded that they are prone to morphological changes with ageing, a warning that the physiology of oligodendrocytes may be eventually affected.
\end{abstract}

In this decade, research on oligodendrocytes has mainly been concerned with problems of myelination (PRAYOONWIWAT and RodRIGUEZ, 1993), with biochemical aspects (YIM et al., 1993) and immunological properties (MASSA et al., 1993), and with relationships between oligodendrocytes and other cell types such as astrocytes (RANSOM and KETTENMANN, 1990). Additonally, numerous studies have been done on oligodendrogliogenesis (LEVISON and GOLDMAN, 1993). In strong contrast, fewer studies have been available concerning the ageing process of oligodendrocytes in the adult, either qualitatively (HASAN and GLEES, 1973; DE LA RozA et al., 1985) or quantitatively (LING and LEBLOND, 1973; WENDER et al., 1988).

The aim of this study, therefore, is to seek whether there are qualitative as well as quantitative agerelated morphological changes in oligodendrocytes of the cerebellar granular layer or-because these cells are capable of mitosis (STURROCK and MCRAE, 1980; VICK and DEVRIES, 1992)-whether they do not change with ageing. To the best of our knowledge, no work stating qualitative or quantitative age-related variations on the cerebellar cortex oligodendrocytes has ever been published. The results may predict putative physiological changes, especially in nerve conduction in the central nervous system.

\section{MATERIAL AND METHODS}

\section{Preparation of tissue for transmission electron microscopy}

Female Sprague-Dawley albino rats 2, 6, 9, 12, 15, 18, 21 and 24 months old ( $n=5$ per age) were perfused through the heart with $2.5 \%$ glutaraldehyde in $0.1 \mathrm{M}$

*This study was partially supported by a research contract from JNICT, Lisbon, Portugal (Project PBIC/C/CEN/1114/ 92) and a grant from the Eng. António de Almeida Foudation (Oporto, Portugal). 
Sørensen's phosphate buffer ( $\mathrm{pH}$ 7.3) after being anaesthetized with $3.5 \%$ aqueous solution of chloral hydrate $(35 \mathrm{mg}$ per $100 \mathrm{~g}$ of body weight, intraperitoneally). Sucrose was added to $0.1 \%$ to the final fixative solution. Samples from the neocerebellum (Crus I and Crus II) were removed and processed for conventional electron microscopy as described in a previous study (MONTEIRO, 1989). The sections were observed under an electron microscope JEOL JEM $100 \mathrm{CXII}$, operated at $60 \mathrm{kV}$.

\section{Morphometric analysis}

To obtain randomisation, samples of tissue were haphazardly chopped into tiny pieces $\left(<1 \mathrm{~mm}^{3}\right)$ during fixation and, after being subsequently processed, they were embedded in resin with no special orientation. Only one single cell profile from each randomly chosen block was selected and photographed at a primary magnification of $\times 5,300$ : this was the first one to appear in the electron microscopic field, disregarding either the shape or size of the profile. No serial sectioning was carried out.

A multipurpose test system, widely known as a standard morphometric technique (WEIBEL and BOLENDER, 1973), with 84 lines of $1.35 \mathrm{~cm}$ lengths enabled the determination on prints (magnified $\times 15,900$ ) of the volume density or fractional volume of the nucleus $(\mathrm{Vv}$, expressed as a \%) relative to the whole oligodendrocyte soma:

$$
\mathrm{Vv} \text { (nucleus, soma })=(\mathrm{P} / \mathrm{Pt}) \cdot 100
$$

where $P$ is the number of test points laying over the nucleus and $\mathrm{Pt}$ is the total number of points laying over the reference space. A total of 640 oligodendrocyte somatic profiles were analysed ( 80 per age group, 16 per animal).

An identical formula also enabled the calculation of the percentages of each type of oligodendrocyte out of the total number of oligodendrocytes counted, where $\mathrm{P}$ is the number of a certain type of oligodendrocyte and $\mathrm{Pt}$ is the total number of oligodendrocytes counted. For this purpose, 100 grids per age were scanned, i.e., 20 per animal. Only one profile of oligodendrocyte (sectioned through the nucleus) per grid was considered; this was again the first one to appear in the electron microscopic field.

\section{Statistical analysis}

The volume density for each individual animal (the mean obtained from the 16 prints), the inter-individual mean ( $\mathrm{n}=5$ animals), the respective standard deviation $(\mathrm{SD})$ and coefficient of variation $(\mathrm{CV}=\mathrm{SD} /$ Mean) are displayed in Table 1, as recently presented by stereologists (MAYHEW, 1989; OSTER et al., 1993: AugsBurger et al., 1993). The percentages of oligodendrocytes with ageing are summarized in Table 2. Because they were estimated only in order to seek a possible correlation with age, the mean value per age is thus the sole number presented. Computer-assisted programmes were used for studying: 1) the one-way analysis of variance or ANOVA (Microsoft Excel, version 4.0 ); 2) the regression analysis (with concomitant analysis of variance) as well as pair comparisons with the Student's t-test (STATGRAPHICS, version $4.0)$; and 3) the graphic representation of the linear trend volume density versus age (HARVARD GRAPHICS, version 2.10). The statistical results were considered not significant when $\mathrm{P}>0.05$.

\section{RESULTS}

\section{General ultrastructure of oligodendrocytes}

The neocerebellar cortex oligodendrocytes (Figs. 1-3) are quite chromophilic elements, usually displaying an eccentric nucleus occupying a large part of the cell and presenting a clearly visible perinuclear cisterna. The cisternae of rough endoplasmic reticulum are generally short, dilated and filled with electron-lucent contents. Ribosomes, either free or aggregated into rosettes are abundant; together with a prolific population of microtubules and an inherent chromophilia of the cytoplasmic matrix, they are the elements responsible for the characteristic cytoplasmic electron-density. All typical, osmiophilic and most pleomorphic dense bodies are constant (Fig. 1).

Fig. 1. Type I oligodendrocyte. Note the smooth contour of the nucleus $(N)$, which exhibits a typically dilated perinuclear cisterna. In the cytoplasm may be seen dense bodies (arrows) as well as short and distended cisternae of rough endoplasmic reticulum (circles) filled with electron-lucent contents. $M$ myelinated axons. (Rat age $=18$ months). $\times 18,000$

Fig. 2. Type II oligodendrocyte with the nucleus $(N)$ exhibiting a tortuous contour, dilated perinuclear cisterna and very dense heterochromatin masses, strongly contrasting with the euchromatin. Note the very dark tonality of the cytoplasmic matrix, where clusters of free ribosomes may be seen; in addition, typical short and dilated cisternae of rough endoplasmic reticulum with clear contents stand out (arrows). Asterisk Golgi apparatus, circle nuclear pore, $M$ myelinated axon. (Rat age $=24$ months). $\times 24,000$ 




Figs. 1 and 2. Legends on the opposite page. 




Fig. 3. Type II oligodendrocyte with a nucleus $(N)$ exhibiting a tortuous contour and dilated perinuclear cisterna. Amidst the karyoplasm, an inclusion of loose, wavy and non-crystalline filaments stands out (arrows). $G$ granule cell. (Rat age $=21$ months). $\times 24,000$

Fig. 4. A close-up of the filaments (arrows) noticed in the oligodendrocyte profile of Fig 3. $\times 60,000$ 


\section{Types of oligodendrocytes}

Two types of oligodendrocytes are distinguished: Type I (Fig. 1) and Type II (Figs. 2, 3). In the former, the nucleus is spherical or elliptical, showing a very smooth contour. Compared with Type I, Type II oligodendrocytes are much darker, exhibiting a nucleus with inconsistent form and with a more tortuous outline, as well as showing a wider perinuclear cisterna. The contrast between euchromatin and heterochromatin is usually most prominent. In addition, clearly longer cisternae of rough endoplasmic reticulum are much more common, and the Golgi complex is more conspicuous.

\section{Nuclear filaments}

From 18 months onwards, and with increasing frequency, inclusions occurring as bundles of loose, wavy and non-crystalline nuclear filaments appear amidst the euchromatin zones (Figs. 3, 4). The filaments do not follow a straight course (in fact, some elements seem to intersect); nonetheless, they are oriented along the length of the euchromatin zone which contains them and where the karyoplasmic matrix seems to be more delicate. They are long, slender, unbranched and indefinite in length; only one bundle per profile was detected, consisting of a variable number of filaments. In addition, their occurrence only attains Type II oligodendrocytes. The filaments, which are much narrower than the microtubules, measure $10.9 \pm 3.1 \mathrm{~nm}($ Mean $\pm \mathrm{SD})$ in thickness.

\section{Volume density of the nucleus}

The values of $\mathrm{Vv}_{\mathrm{v}}$ of the nucleus (Table 1) reveal that the parameter presents a variation with ageing. The minimum one is obtained at 2 months, being statistically significant from the values of any of the other ages. Either the maximum value (at 21 months) or the second highest one (at 24 months) are significant from those occurring at 2, 6 and 15 months. The CVs obtained in our study are excellent in statistical terms (the highest one is $11 \%$ ); these, together with the very low coefficients of error $(\mathrm{CE}=\mathrm{CV} / \sqrt{\mathrm{n}})$ indicate that the biological variation within each age is low and that the sampling scheme we used was highly efficient. The one-way analysis of variance (ANOVA)

Table 1. Volume densities $\left(\mathrm{V}_{\mathrm{v}}-\%\right)$ of the nucleus of oligodendrocytes of the granular layer of the rat neocerebellar cortex (Crus I and Crus II), from 2 to 24 months

\begin{tabular}{lcccccccc}
\hline Age (months) & 2 & \multicolumn{1}{c}{6} & \multicolumn{1}{c}{9} & \multicolumn{1}{c}{12} & \multicolumn{1}{c}{15} & 18 & \multicolumn{1}{c}{21} & \multicolumn{1}{c}{24} \\
\hline Rat 1 & 46.09 & 57.70 & 51.74 & 59.65 & 52.99 & 61.75 & 64.89 & 61.65 \\
Rat 2 & 49.86 & 53.73 & 53.32 & 65.96 & 55.75 & 54.53 & 70.60 & 62.22 \\
Rat 3 & 51.83 & 59.51 & 60.69 & 62.18 & 61.51 & 64.72 & 69.79 & 66.15 \\
Rat 4 & 52.47 & 58.70 & 64.37 & 58.60 & 61.74 & 61.48 & 59.13 & 69.42 \\
Rat 5 & 53.08 & 62.50 & 65.14 & 55.04 & 57.96 & 66.30 & 60.25 & 59.69 \\
\hline Mean (n=5) & 50.67 & 58.43 & 59.05 & 60.29 & 57.99 & 61.76 & 64.93 & 63.83 \\
SD & 2.83 & 3.18 & 6.21 & 4.08 & 3.76 & 4.52 & 5.28 & 3.91 \\
CV & 0.06 & 0.05 & 0.11 & 0.07 & 0.06 & 0.07 & 0.08 & 0.06 \\
\hline
\end{tabular}

Analysis of pair comparisons: $2 / 6 \mathrm{M}(\mathrm{P}=0.0035) ; 2 / 9 \mathrm{M}(\mathrm{P}=0.0251) ; 2 / 12 \mathrm{M}(\mathrm{P}=$ $0.0025) ; 2 / 15 \mathrm{M}(\mathrm{P}=0.00829) ; 2 / 18 \mathrm{M}(\mathrm{P}=0.00164) ; 2 / 21 \mathrm{M}(\mathrm{P}=0.0007) ; 2 / 24 \mathrm{M}(\mathrm{P}=$ $0.00029) ; 6 / 21 \mathrm{M}(\mathrm{P}=0.045) ; 6 / 24 \mathrm{M}(\mathrm{P}=0.043) ; 15 / 21 \mathrm{M}(\mathrm{P}=0.043) ; 15 / 24 \mathrm{M}(\mathrm{P}=$ $0.042)$.

Table 2. Variations with ageing of crude percentages (means) of Type I oligodendrocytes (ODCs) and of Type II ODCs of the rat neocerebellar cortex (Crus I and Crus II), from 2 to 24 months

\begin{tabular}{lrccccccc}
\hline Age (months) & 2 & 6 & 9 & 12 & 15 & 18 & 21 & 24 \\
\hline Type I ODCs (\%) & 93 & 83 & 80 & 75 & 73 & 50 & 26 & 26 \\
Type II ODCs (\%) & 7 & 17 & 20 & 25 & 27 & 50 & 74 & 74 \\
\hline
\end{tabular}


exhibits an extremely high significant value $(\mathrm{P}<$ $0.0005)$, disclosing a real change in $\mathrm{Vv}$ with ageing. In addition, a high degree of positive linear correlation is detected between the $\mathrm{Vv}$ and age (Fig. 5).

\section{Increase in the number of Type II oligodendrocytes}

For the purpose of seeking a correlation with ageing, the percentages of each type of oligodendrocyte were calculated (Table 2). Results show an increase in the percentage of Type II oligodendrocytes with ageing, which constitutes an extremely significant positive linear trend $[\mathrm{r}=0.943, \mathrm{~F}(1,6)=48.5499, \mathrm{P}=0.00043]$.

\section{DISCUSSION}

\section{Identification of oligodendrocytes}

The general electron microscopic features of oligodendrocytes have been previously reported by many investigators either in the cerebellar cortex or elsewhere (MONTEIRO, 1983). Thus, our present description of oligodendrocytes is only a résumé for bringing forward the matter in consideration, and to avoid mistaking that well defined glial entity for another one, also with dark aspect under the electron microscope, i.e., interstitial microgliacytes. It must be emphasized that, with some degree of expertise, the presence of a nuclear profile in electron micrographs with proper magnification is not necessary for recognizing either the oligodendroglia or other glial cells. In fact, the cytoplasmic characteristics and other additional features-for instance, the connection of oligodendrocytes with myelinated axons, the lack of somatic synapses as well as of Nissl bodies in glial cells-are sufficient elements for their cytological diagnoses. Further details for differential analysis were reported by PALAY and CHAN-PALAY (1974). The nuclear morphology, however, helps the unequivocal separation of oligodendrocytes into two categories; this is why the nucleus was used as a marker for the countings which preceded the percentage estimations.

\section{Oligodendrocyte variability}

Apart from a basic microanatomical description, one must be aware of a certain structural variability among oligodendrocytes. This includes several electrondensities depicted in this study and previously in some others (e.g., KRUGER and MAXWELL, 1967; MORI

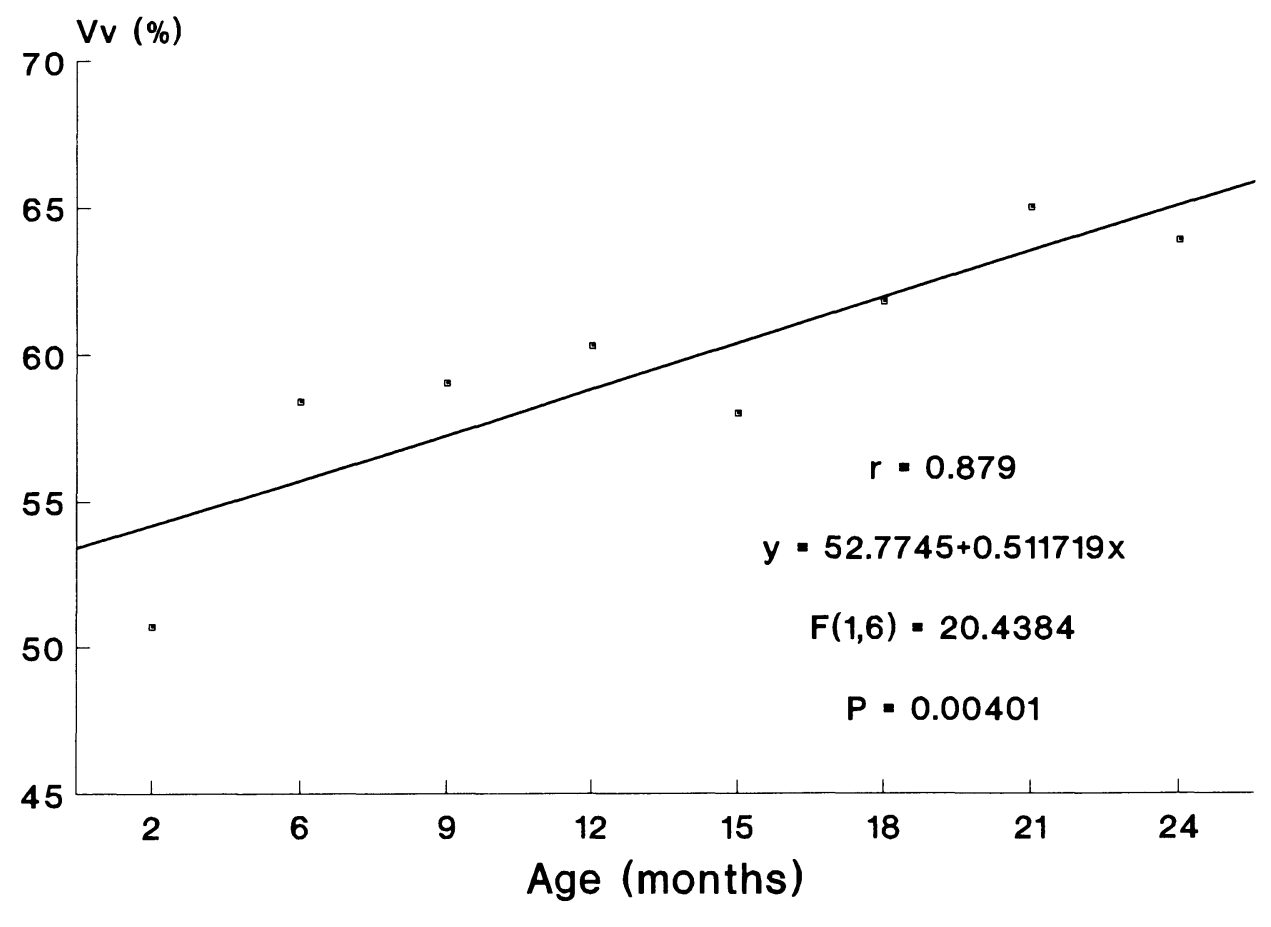

Fig. 5. Age-related changes in the mean volume density (Vv) of the nucleus of oligodendrocytes of the granular layer of the rat neocerebellar cortex (Crus I and Crus II), from 2 to 24 months. 
and LEBLOND, 1970; LING et al., 1973; STENSAAS, 1977; Monteiro, 1983; Monzon-Mayor et al., 1990). Moreover, a "familiar" link, i.e., a developmental sequence, has been recognized from the relatively immature, clear oligodendrocytes to the long lasting, mature, darker oligodendrocytes, with medium-dark oligodendrocytes in between (MORI and LEBLOND, 1970; LING et al., 1973; LING and LEBLOND, 1973; IмАмото et al., 1978). We found two well stated classes of oligodendrocytes, previously designated as Type I and Type II (MonTEIRO, 1983), which correspond, respectively, to the medium-dark and dark types described by the above mentioned authors. In fact, perhaps because we did not include young postnatal animals, oligodendrocytes described as clear by other authors did not appear in our studies.

The relative preponderance in rats of dark forms to lighter ones was seen in cerebral cortex (LING and LEBLOND, 1973) and in the corpus callosum (LING and LEBLOND, 1973; STURROCK, 1976a) from the first month of age, and in the auditory cortex of rats aged 3 months onwards (VAUGHAN and PETERS, 1974). Compared with those results, we observed that in the cerebellar cortex the preponderance is attained at a much later period, i.e., from 21 months onwards.

As mentioned, the percentage of Type II oligodendrocytes increases linearly and significantly with ageing. It must be said, however, that dark oligodendrocytes are smaller cells, a fact seen in other nervous regions (LING et al., 1973; LING and LEBLOND, 1973). Because Abercrombie's correction (ABERCROMBIE, 1946) for nuclear size was not carried out in our study, it may be that the percentages of dark cells out of the total oligodendrocyte population are somewhat underestimated, underscoring that higher numbers are expected to occur. In pragmatic terms, whatever the real unbiased numbers might be, the essential matter is that there is an increase in dark form numbers throughout the ageing process.

Furthermore, it should be said that the mean nuclear diameters (D) of Type I and of Type II oligodendrocytes are quite similar; at 2 months, for instance, $7.8 \mu \mathrm{m}$ and $6.9 \mu \mathrm{m}$ are respectively found, giving a ratio $\left(\mathrm{D}_{\text {II }} / \mathrm{D}_{\mathrm{I}}\right)$ of 0.885 . That diameter may be deduced with a formula which deals with the average "diameters" measured in the profiles (WEIBEL and BOLENDER, 1973). Despite the variation in nuclear size throughout ageing, the above mentioned ratio seems to keep practically steady; at 15 months, for example, $6.9 \mu \mathrm{m}$ and $6.2 \mu \mathrm{m}$ are respectively estimated, with a ratio of 0.899 . Thus, again, the omission of Abercrombie's correction for nuclear size of the oligodendrocytes does not seem to raise any methodological problem.
In spite of the presence of two types of oligodendrocytes, we did not perform a differential morphometric analysis because they are, as a whole, a well defined glial entity. Secondly, as the two types are linked in early postnatal differentiation, they are most probably by analogy also connected in older animals, although at slower rate. Our set of studies on the ultrastructure of the rat neocerebellar cortex (e.g., MonTEIRo 1983, 1986, 1989, 1991a, b; MonTEIRO et al., 1992a, b, 1994) never detected any unequivocal mitotic figure in oligodendrocytes, which correlates with their very low mitotic index in normal adult animals (SKOFF et al., 1976; KORR, 1980; KAPLAN and HINDS, 1980). Therefore, the positive linear trend perceived when the percentage of Type II oligodendrocytes is plotted against age would be the result of a progressive differentiation from Type I to Type II oligodendrocytes.

Furthermore, cells with the structure of the immature glioblastic/oligodendroblastic lineage were never depicted in the age spectrum we used; as is widely known, they are mitotic elements (IмAмото et al., 1978), and according to a number of authors, they persist in adult life in very small percentages (LING and LeBlOND, 1973; STURROCK, 1976a, b). In summary, therefore, because the two types do not seem to undergo a separate age-related evolution, it would be artificial to divide them into two groups.

\section{Nuclear filaments as an age-related feature in oligodendrocytes}

Under the qualitative point of view, the only ultrastructural feature that seems an obvious age-dependent modification is the presence of the inclusions of nuclear filaments described which appear with increasing frequency from 18 months onwards. The estimated dimensions of the filaments permit their classification as intermediate; however, they do not resemble at all the typical glial filaments (undoubtedly of the intermediate kind) seen in the other type of macroglia, i.e., the astroglia. To the best of our knowledge, no other author has mentioned filaments in the karyoplasm of oligodendrocytes; nonetheless, crystalline inclusions have been found either in the perinuclear cisterna or in the cisternae of rough endoplasmic reticulum (MARQUET and SOBEL, 1969).

The so-called "hyaline globules" as well as the "granular deposits", inclusions perceived in the cytoplasm of astrocytes (MONTEIRO et al., 1992a) and curiously also seen from 18 months onwards, were never noticed in oligodendrocytes. In turn, the circular membrane-bound inclusions displayed by astroglial nuclei during ageing (FIELD and PEAT, 1971) were 
never found in our studies. It is our opinion that the inclusions we saw in oligodendrocytes may be regarded as age-related specific structural markers, indeed very specific when one recalls that they only appear in Type II oligodendrocytes. CostA et al. (1992) also described juxtanuclear inclusions in oligodendrocytes as early and specific markers of multiple system atrophy.

In contrast, age-related nuclear inclusions are common occurrences in neurons noticed since MANN (1894) and successively by a number of authors (JOHNSON and Miquel, 1974; JoHnson et al., 1975; VAUGHAN and VINCENT, 1979; and many others). The nature of the inclusions is still under dispute. As yet, we also are not in a position to advance any hypothesis about the origin and importance of the oligodendroglia nuclear inclusions.

\section{Conclusion}

Although qualitative changes and significant morphometric results were found concerning the $\mathrm{Vv}$ of the nucleus and subtypes of oligodendrocytes, it would be premature to draw linear conclusions as to whether they may interfere with the biochemical machinery of the oligodendrocytes; nonetheless, structural modifications may serve to warn about possible and important physiological implications.

The production and maintenance of the myelin sheath is apparently the only function that can be performed by oligodendrocytes with a well-established certainty. Nevertheless, other activities have been attributed to oligodendrocytes (HU et al., 1987; URADE et al., 1987; OH et al., 1991) such as being putatively important in the control of the nervous homeostasy. If so, it is logical to speculate that oligodendrocyte age-related changes may predict alterations in the level of myelination, and eventually in some other functions. It is important to clarify this with further studies, because demyelination accounts for very distressful diseases such as multiple sclerosis and leukodystrophies.

Acknowledgements. We are greatly indebted to Mrs. M. F. Sieuve-MonTEIRo for correction of the translation of the manuscript.

\section{REFERENCES}

ABERCRoMbie, M.: Estimation of nuclear population from microtome sections. Anat. Rec. 94: 238-247 (1946).

Augsburger, H. R., L. M. Cruz-Orive and S. Arnold: Morphology and stereology of the female canine urethra correlated with the urethral pressure profile. Acta Anat. 148: 197-205 (1993).
Costa, C., C. Duyckaerts, P. Cervera and J.-J. Hauw: Les inclusions oligodendrogliales, un marqueur des atrophies multisystématisées. Rev. Neurol. 148: 274-280 (1992).

De la Roza, C., J. Cano and F. Reinoso-Suárez: An electron microscopic study of astroglia and oligodendroglia in the lateral geniculate nucleus of aged rats. Mech. Ageing Devel. 29: 267-281 (1985).

Field, E. J. and A. Peat: Intranuclear inclusions in neurones and glia: a study in the ageing mouse. Gerontologia 17: 129-138 (1971).

Hasan, M. and P. Glees: Ultrastructural age changes in hippocampal neurons, synapses and neuroglia. Exp. Gerontol. 8: 75-83 (1973).

Hu, Z. Y., E. Bourreau, I. Jung-Testas, P. Robel and E.-E. BAUliEU: Neurosteroids: oligodendrocyte mitochondria convert cholesterol to pregnelone. Proc. Nat. Acad. Sci. 84: 8215-8219 (1987).

Imamoto, K., J. A. Paterson and C. P. Leblond: Radioautographic investigation of gliogenesis in the corpus callosum of young rats. I. Sequential changes in oligodendrocytes. J. Comp. Neurol. 180: 115-138 (1978).

Johnson, J. E. Jr. and J. Miquel: Fine structural changes in the lateral vestibular nucleus of aging rats. Mech. Ageing Devel. 3: 203-224 (1974).

Johnson, J. E. Jr., W. R. Mehler and J. Miquel: A fine structural study of degenerative changes in the dorsal column nuclei of aging mice: lack of protection by vitamin E. J. Gerontol. 30: 395-411 (1975).

KAPLAN, M. S. and J. W. Hinds: Gliogenesis of astrocytes and oligodendrocytes in the neocortical grey and white matter of the adult rat: electron microscopic analysis of light radioautographs. J. Comp. Neurol. 193: 711-727 (1980).

KoRr, H.: Proliferation of different cell types in the brain. Adv. Anat. Embryol. Cell Biol. 61: 1-69 (1980).

Kruger, L. and D. S. Maxwell: Comparative fine structure of vertebrate neuroglia: teleosts and reptiles. J. Comp. Neurol. 129: 115-142 (1967).

Levison, S. W. and J. E. Goldman : Both oligodendrocytes and astrocytes develop from progenitors in the subventricular zone of postnatal rat forebrain. Neuron 10: 201-212 (1993).

Ling, E. A. and C. P. LEblond: Investigation of glial cells in semithin sections. II. Variation with age in the numbers of the various glial cell types in rat cortex and corpus callosum. J. Comp. Neurol. 149: 73-82 (1973).

Ling, E. A., J. A. Paterson, A. Privat, S. Mori and C. P. LEBLOND: Investigation of glial cells in semithin sections. I. Identification of glial cells in the brain of young rats. J. Comp. Neurol. 149: 43-72 (1973).

MANN, G.: Histochemical changes induced in sympathetic motor and sensory nerve cells. J. Anat. 29: 100-108 (1894).

MARquet, E. and H. J. Sobel: Crystalline inclusions in the nuclear envelope and granular endoplasmic reticulum of the fish spinal cord. J. Cell Biol. 41: 774-785 (1969).

Massa, P. T., K. Ozato and D. E. McFarlin: Cell type- 
specific regulation of major histocompatibility complex (MHE) class I gene expression in astrocytes, oligodendrocytes, and neurons. Glia 8: 201-207 (1993).

MaYhew, T. M.: Stereological studies on rat spinal neurons during postnatal development: estimates of mean perikaryal and nuclear volumes free from assumptions about shape. J. Anat. 162: 97-109 (1989).

Monteiro, R. A. F.: Do the Purkinje cells have a special type of oligodendrocyte as satellites? J.Anat. 137: 71-83 (1983).

- Critical analysis on the nature of synapses en marron of cerebellar cortex. J. Hirnforsch. 27: 567-576 (1986).

: Morphometric differences between basket cells and stellate cells of rat neocerebellum (Crus I and Crus II). J. Submicrosc. Cytol. Pathol. 21: 725-736 (1989).

- : Age-related quantitative changes in the organelles of rat neocerebellar Purkinje cells. Histol. Histopathol. 6: 9-20 (1991a).

- Genesis, structure and transit of dense bodies in rat neocerebellar cortical cells, namely Purkinje neurons: an ultrastructural study. J. Hirnforsch. 32: 593-609 (1991b).

Monteiro, R. A. F., E. Rocha and M. M. MariniABREU: Age-related morphometric changes occurring in the somata of astrocytes of the granular layer of rat neocerebellar cortex (Crus I and Crus II). Histol. Histopathol. 7: 427-444 (1992a).

- - : Quantitative age-related changes on nuclear invaginations of neocerebellar Purkinje cells. Neuroreport 3: 1089-1092 (1992b).

- : Heterogeneity and death of Purkinje cells of rat neocerebellum (Crus I and Crus II): hypothetic mechanisms based on qualitative and quantitative microscopical data. J. Brain Res. 35: 205222 (1994).

Monzon-Mayor, M., C. YAnes, J. L. James and R. R. STURRock: An ultrastructural study of the development of oligodendrocytes in the midbrain of the lizard. J. Anat. 170: 43-49 (1990).

MoRI, S. and C. P. LEblond: Electron microscopic identification of three classes of oligodendrocytes and a preliminary study of their proliferation activity in the corpus callosum of young rats. J. Comp. Neurol. 139: 1-30 (1970).

OH, Y. J., G. J. Markelonis and T. H. OH: Immunocytochemical localization of mitochondrial malate dehydrogenase in primary cultures of rat astrocytes and oligodendrocytes. J. Histochem. Cytochem. 39: 681-688 (1991).

Oster, S., P. Christoffersen, H. J. G. Gundersen, J. O. Nielsen, B. Pakkenberg and C. Pedersen: Cerebral atrophy in AIDS: a stereological study. Acta Neuropathol. 85: 617-622 (1993).

Palay, S. L. and V. Chan-Palay: Cerebellar cortex. Cytology and organization. Springer-Verlag, BerlinHeidelberg-New York, 1974.

Prayoonwiwat, N. and M. Rodriguez: The potential for oligodendrocyte proliferation during demyelinating disease, J. Neuropathol. Exp. Neurol. 52: 55-63 (1993).

Ransom, B. R. and H. Kettenmann : Electrical coupling, without dye coupling, between mammalian astrocytes and oligodendrocytes in cell culture. Glia 3: 258-266 (1990).

Skoff, R. P., D. L. Price and A. Stocks: Electron microscopic autoradiographic studies of gliogenesis in rat optic nerve. I. Cell proliferation. J. Comp. Neurol. 169: 291-311 (1976).

STEnsaAs, L. J.: The ultrastructure of astrocytes, oligodendrocytes, and microglia in the optic nerve of urodele amphibians (A. punctatum, T. pyrrhogaster, $T$. viridescens). J. Neurocytol. 6: 269-286 (1977).

STURRock, R. R.: Light microscopic identification of immature glial cells in semithin section of the developing mouse corpus callosum. J. Anat. 122: 521-537 (1976a).

- Changes in neuroglia and myelination in the white matter of aging mice. J. Gerontol. 31: 513-522 (1976b).

STURRock, R. R. and D. A. MCRAE: Mitotic division of oligogendrocytes which have begun myelination. J. Anat. 131: 577-582 (1980).

Urade, Y., N. Fujimoto, T. Kaneko, A. Konishi, N. Mizuno and O. Hayashi: Postnatal changes in the localization of prostaglandin D synthetase from neurons to oligodendrocytes in the rat brain. J. Biol. Chem. 262: 15132-15136 (1987).

Vaughan, D. W. and A. Peters: Neuroglial cells in the cerebral cortex of rats from young adulthood to old age: an electron microscope study. J. Neurocytol. 3: 405-429 (1974).

VAughan, D. W. and J. M. VincenT: Ultrastructure of neurons in the auditory cortex of ageing rats: a morphometric study. J. Neurocytol. 8: 215-228 (1979).

VICK, R. S. and G. H. DEVRIES: Mitotic potential of adult rat oligodendrocytes in culture. J. Neurosci. Res. 33: 6874 (1992).

Weibel, E. R. and R. P. Bolender: Stereological techniques for electron microscopy morphometry. In: (ed. by) M. A. HAyat: Principles and techniques of electron microscopy. Biological applications. Van Nostrand Reinhold Company, New York, 1973 (Vol. 3, p. 237-296).

Wender, M., J. Szczech, A. Godlewski and A. Groch owALSKA: Karyometric and cytophotometric studies of the oligodendroglia in the corpus callosum of the rat after hypoxia. Exp. Pathol. 33: 249-255 (1988).

YIM, S. H., J. E. SHERIN and S. Szuchet: Oligodendrocyte proteoglycans: modulation by cell-substratum adhesion. J. Neurosci. Res. 34: 401-413 (1993).

Prof. Rogério Monteiro

Laboratory of Histology and Embryology

Institute of Biomedical Sciences

Largo Professor Abel Salazar, no. 2

4050 Porto, Portugal 